\title{
Health promotion by the dental team
}

General health promotion in general dental practice - The involvement of the dental team

Part 1: A review of the evidence of effectiveness of brief public health interventions

T. A. Dyer and P. G. Robinson BrDent J 2006; 200: 679-685

\section{Objectives}

To review the evidence of the effectiveness of dentists, dental teams and other healthcare workers in seven different brief public health interventions that might contribute to Government targets in cancer and circulatory disease. The interventions were: smoking prevention, smoking cessation, advice on alcohol consumption, diet counselling, advice on physical exercise, advice on skin cancer prevention and blood pressure monitoring.

Method

A series of literature reviews, using a generic systematic approach, were undertaken to investigate the effectiveness of dentists, dental teams and other healthcare workers in each intervention.

Results

Apart from smoking cessation and dietary advice, no studies were identified on the effectiveness of dentists or dental teams in the interventions investigated. There is some evidence that dentists and dental teams can be effective in smoking cessation. There is minimal evidence for effectiveness in dietary counselling, and that which exists shows only a transient effect. There is evidence that other healthcare workers can have some effect in all interventions, though the effect in preventing skin cancer is questionable.

Conclusions

Due to the paucity of studies undertaken, there is minimal evidence of effectiveness of dentists and dental teams in any of the seven interventions. However other healthcare workers are effective in most of them. Dentists and dental teams' involvement in such brief general health promotion interventions might contribute to Government targets on cancer and circulatory disease.

\section{IN BRIEF}

- UK Government policy aims to improve health by reducing levels of chronic disease, especially coronary heart disease, obesity and cancer.

- Dental teams could contribute to Government targets set for reducing these diseases through preventive health interventions.

- Current health policy emphasises evidence-based commissioning of services.

- Dental teams' future involvement in general health promotion will depend on whether the profession sees itself as having a general public health role and whether it is economically viable for them to do so.

\section{COMMENT}

Government policy is increasingly emphasising the importance of expanding public health activity within and beyond the NHS. Although the role of prevention in promoting oral health is well recognised, these opportunities have not been fully used by the dental profession. Encouraging dental practitioners to become actively engaged in a wider range of actions aimed at preventing other systemic chronic diseases is therefore a major challenge. A core principle underpinning public health practice is the need to address the common risks to health based upon a contemporary evidence base. The most appropriate methodology to assess the effectiveness of public health interventions is however, a highly contested issue.

This first of two papers investigating the potential role of dental practitioners in a range of preventive actions assesses the evidence base underpinning each activity. Based upon a hierarchy of evidence used in evidence based medicine, Dyer and Robinson have reviewed the effectiveness of the following preventive interventions: smoking prevention; smoking cessation; advice on alcohol consumption; diet counselling; advice on physical exercise; advice on skin cancer prevention and blood pressure monitoring. Their review sought to determine the evidence base for these brief interventions undertaken in primary care settings by health professionals, including dentists and PCDs. The most striking result from this review is the absence of evidence on the effectiveness of dental practitioners and PCDs in these areas of preventive practice. Major gaps in research have therefore been highlighted by this review. However as the authors conclude, encouraging evidence does exist for other health professionals. A potential therefore exists for developing a wider role for dentists and PCDs in chronic disease prevention.

Any attempt at changing clinical practice requires more than a summary of the supporting evidence. As the authors rightly highlight research is needed to assess the views and experiences of dental practitioners to determine how best to support them to adopt a more preventive style of practice. This will be the focus of the next paper in this short series. In addition, a move to a more preventive approach will only occur when the system of reimbursing dentists is reformed and appropriate incentives are provided to encourage evidence based prevention. It remains to be seen whether the new dental contract in England will facilitate this change or not.

R. G. Watt, Professor in Dental Public Health, University College London

DOI: 10.1038/sj.bdj.4813732 